\title{
Desenvolvimento da Memória na Criança: O que Muda com a Idade?
}

\author{
Memory Development in Children: What Changes with Age?
}

\author{
Maria Paula Carneiro* \\ Universidade Lusófona de Humanidades e Tecnologias, Lisboa, Portugal
}

\begin{abstract}
Resumo
Neste artigo é apresentada uma revisão da literatura sobre o desenvolvimento da memória, organizada de acordo com a divisão dos diferentes armazenamentos ou sistemas existentes. O desenvolvimento das memórias sensorial, de trabalho, explícita e implícita é analisado desde o período pré-escolar. Embora ocorram mudanças significativas na memória de trabalho e explícita, parece não existir evolução da memória implícita perceptiva ao longo do desenvolvimento.

Palavras-chave: Desenvolvimento; memória; sistemas de memória; crianças.
\end{abstract}

\begin{abstract}
This article presents a review of the literature about memory development, organized according to the different storages or memory systems. The development in sensory memory, working memory, explicit memory and implicit memory is analysed from the preschool period on. Although significant changes take place in the working and explicit memory, the perceptual implicit memory remains invariant with age.

Keywords: Development; memory; memory systems; children.
\end{abstract}

Com este artigo pretende-se abordar o tema do desenvolvimento da memória, tendo por base algumas das distinções efectuadas entre diferentes armazenamentos ou sistemas de memória, as quais provêm sobretudo de evidência experimental e neuropsicológica. Foi, assim, objetivo desta revisão da literatura analisar em que sistemas ocorrem modificações da memória com a idade e, nesses casos, descrever a sua evolução. Esta análise iniciou no período pré-escolar, o qual já permite a utilização de um procedimento experimental baseado numa resposta verbal e atribuiu especial ênfase à passagem do período préescolar para o período escolar.

A existência de diversas nomenclaturas relativas às divisões da memória coloca-nos perante a dificuldade de decisão quanto a qual adoptar para caracterizar o desenvolvimento de cada tipo de memória. Para compreensão das várias terminologias, as quais serão posteriormente usadas ao longo do texto, é realizada uma breve síntese da origem das diferentes taxonomias que têm sido adoptadas no estudo das divisões da memória.

Uma das mais antigas divisões da memória reside na distinção entre memória sensorial, memória a curto prazo e memória a longo prazo, a qual advém do modelo de multiarmazenamento proposto por Atkinson e Shiffrin (1968).

Este artigo foi baseado em partes da dissertação de doutorado do autor. Agradece-se a contribuição dos orientadores Pedro Albuquerque e Angel Fernandez e as sugestões de dois revisores anônimos.

${ }^{*}$ Endereço para correspondência: Campo Grande, 376, 1749024, Lisboa, Portugal. E-mail:mpcarneiro@gmail.com
Segundo este modelo, cada tipo de memória representa um determinado armazenamento existente num estádio específico do processamento da informação. A informação é recebida, em primeiro lugar, no armazenamento sensorial, onde é mantida durante poucos segundos ou fracções de segundo após o desaparecimento do estímulo. Seguidamente passa para o armazenamento a cur to prazo, o qual retém apenas uma determinada quantidade de informação durante menos de 1 minuto. Depois de passar pelo armazenamento a curto prazo a informação ou é esquecida ou, se for processada, por exemplo, através da recapitulação, pode passar para o armazenamento a longo prazo, onde pode permanecer indefinidamente nesse compartimento de capacidade ilimitada.

Porém, com Baddeley e Hitch (1974) esta concepção de memória a curto prazo, encarada como um compartimento de armazenamento temporário, foi modificada através do modelo de memória de trabalho de maneira a explicar a manutenção temporária da informação enquanto são executadas operações mentais. De acordo com este modelo a informação é temporariamente mantida durante a execução de determinadas operações mentais, podendo ser classificada, organizada e relacionada com outra informação que já se encontra retida na memória. Segundo a maior parte dos autores, os diferentes componentes do modelo da memória de trabalho fornecem uma vantagem na explicação de tarefas cognitivas que incluem um sistema de manipulação temporária da informação, tais como a leitura, a matemática, o raciocínio e a resolução de problemas (Baddeley, 1986). 
É na generalidade aceite que a memória a longo prazo não é um sistema unitário, mas que possui alguma organização de forma a lidar com a enorme quantidade de informação que os indivíduos possuem. A primeira distinção dentro da memória a longo prazo foi proposta por Tulving (1972) e incluiu dois sistemas, a memória semântica e a memória episódica. A memória semântica representa o armazenamento do conhecimento geral acerca do mundo, relacionado com o significado das palavras e conceitos, enquanto que a memória episódica refere-se ao armazenamento de acontecimentos pessoais ocorridos num lugar e num tempo particulares. Mais tarde, em 1985, Tulving incluiu uma forma adicional de memória designada de memória procedimental. Este tipo de memória envolve a aquisição de conhecimento que não está disponível através de uma averiguação consciente, incluindo a capacidade para aprender hábitos, competências, priming e algumas formas de condicionamento clássico. De acordo com Tulving (1985), estes três sistemas de memória são caracterizados por níveis distintos e hierárquicos de consciência: a memória procedimental fornece a consciência anoetica, ou seja a possibilidade de reagir a estímulos internos e externos; a memória semântica é caracterizada pela consciência noetica ou introspecção consciente do mundo externo e interno; e a memória episódica fornece a consciência autonoetica, responsável pelo auto-conhecimento e auto-consciência.

Como a distinção entre memória semântica e episódica não foi inteiramente apoiada pela observação de alguns pacientes amnésicos (Parkin, 1997), Cohen e Squire (1980) numa tentativa de ultrapassar este problema, propuseram o conceito de memória declarativa, a qual inclui ambos os conhecimentos semântico e episódico proposto por Tulving (1972) e é definida como uma memória que pode ser acedida conscientemente e que corresponde ao "saber que”. A memória considerada não declarativa foi definida de forma similar à memória procedimental de Tulving (1985) e corresponde ao "saber como". Os autores desta distinção de memória sugeriram que os amnésicos possuem uma deterioração geral da memória declarativa mas uma memória procedimental intacta.

Posteriormente, Graf e Schacter, em 1985 e Schacter, em 1987, definiram pela primeira vez os conceitos de memória implícita e explícita, embora autores anteriores a estes, como por exemplo, Claparede, Freud e Korsakoff (citados por Schacter, 1987), já tivessem reportado várias observações de memória implícita. Esta taxonomia não se refere propriamente a sistemas de memória mas incide sobretudo na experiência psicológica durante o momento de recuperação de informação, numa tentativa de explicar como a memória a longo prazo responde a testes de memória explícitos e implícitos. A memória é considerada explícita quando o desempenho de uma tarefa requer uma recordação consciente de experiências prévias e pode ser directamente testada através de tarefas de evocação livre, evocação guiada e reconhecimento (se bem que a tarefa de reconhecimento nem sempre é considerada exclusivamente explícita, ver Gardiner, 1988; Gardiner \& Java, 1993;
Yonelinas, 2002). Por sua vez a memória é considerada implícita quando experiências prévias facilitam o desempenho em determinada tarefa que não requer uma recuperação intencional e consciente dessas experiências. As tarefas mais usadas para avaliação da memória implícita têm sido as de identificação perceptiva, tais como as tarefas de identificação de fragmentos de palavras ou figuras e de completamento de palavras. Correntemente a memória implícita é avaliada através da facilitação do processamento de um estímulo em função da apresentação prévia do mesmo estímulo ou associado, fenómeno conhecido por efeito de priming. À semelhança da distinção entre memória declarativa e não declarativa, o estudo dos pacientes amnésicos tem apoiado esta distinção entre memória explícita e implícita. No geral, quando testados através de tarefas explícitas os amnésicos evidenciam uma memória pobre, no entanto, quando são testados por tarefas implícitas, demonstram níveis de funcionamento semelhantes aos de participantes do grupo controlo (para uma revisão destes estudos ver Roediger \& McDermott, 1993). Apesar de terem tido diferentes origens, os termos memória explícita e declarativa são presentemente usados indistintamente pela maior parte dos autores, da mesma forma que o termo memória implícita é por vezes referido como memória não declarativa ou procedimental.

Com o intuito de fornecer uma descrição organizada do desenvolvimento da memória partimos dos três grandes sistemas de memória que integram a maior parte dos modelos teóricos: a memória sensorial, a memória a curto prazo e a memória a longo prazo. Dentro da memória a longo prazo foi ainda considerada a divisão em memória declarativa ou explícita e não declarativa ou implícita, de acordo com as taxonomias anteriormente descritas.

\section{Desenvolvimento nos Diferentes Armazenamentos ou Sistemas de Memória}

\section{Memória Sensorial}

A informação que chega ao indivíduo é primeiramente mantida pelos registos sensoriais durante fracções ou escassos segundos após o desaparecimento do estímulo. Esta forma de armazenamento sensorial para material visual é denominada de ícone e foi em primeiro lugar demonstrada experimentalmente por Sperling em 1960 (citado por Parkin, 1997). Este autor apresentou a participantes adultos nove letras, dispostas em três linhas, numa matriz $3 \mathrm{x}$ 3 , por um período de tempo muito breve (50 milésimos de segundo). A tarefa dos participantes consistia em evocar o conjunto de letras imediatamente após a sua apresentação. Observou-se que, nesta condição, os participantes não conseguiam nomear mais do que quatro ou cinco letras, pois o ícone decaía muito rapidamente. Contudo, numa outra condição em que era utilizado um procedimento de relato parcial, a evocação correcta chegava aos $100 \%$. Nesta condição os participantes eram informados, através de um sinal e imediatamente depois da apresentação do estímulo, sobre qual a linha de letras a evocar. O sucesso de executar esta tarefa pela maioria dos participantes, na maior parte 
dos ensaios, sugere a existência de um registo visual que comporta determinada quantidade de informação. Mesmo intervalos muito curtos entre o desaparecimento do estímulo e a ocorrência do sinal produziam uma diminuição significativa na quantidade de informação evocada, confirmando que a memória icónica é de muito curta duração (ex., $250 \mathrm{~ms}$ ).

Estudos subsequentes que utilizaram variantes da técnica de relato parcial com crianças (Hoving, Spencer, Robb, \& Schulte, 1978; Sheingold, 1973) mostraram diferenças etárias mínimas na evocação dos itens quando o intervalo entre o desaparecimento do estímulo e a ocorrência do sinal era muito curto, o que sugere uma capacidade do armazenamento sensorial icónico semelhante ao dos adultos. Por sua vez, com intervalos mais longos, as diferenças etárias tornavam-se significativas, evidenciando diferenças na quantidade de informação que é transferida para a memória a curto prazo. Foram mencionados dois tipos distintos de explicação. Por um lado, pode-se considerar que as crianças mais novas transferem menos informação para a memória a curto prazo do que as crianças mais velhas e adultos porque são menos hábeis a utilizar estratégias de codificação verbal, recapitulação visual e de focalização da atenção em partes específicas do ícone. Por outro lado, como o ícone decai rapidamente, é provável que a menor velocidade com que a informação é processada nas crianças mais novas leve a que menor quantidade de informação seja transferida para a memória a curto prazo (Schneider \& Bjorklund, 1998).

\section{Memória a Curto Prazo ou Memória de Trabalho}

A capacidade limitada da memória a curto prazo conter informação é designada de amplitude de memória e habitualmente é medida pela técnica de memória para dígitos que consiste em repetir, pela mesma ordem, os dígitos apresentados, imediatamente após os ter ouvido ou visto. Em média a amplitude de memória para dígitos de um adulto é de aproximadamente $7 \pm 2$ itens (Miller, 1956). Estudos desenvolvimentais que usaram este tipo de tarefa evidenciaram diferenças etárias ao nível da amplitude da memória (para uma revisão destes estudos ver Dempster, 1981), aumentando esta gradualmente com a idade. Enquanto que aos 2 anos a amplitude de memória é de aproximadamente 2 itens, aos 9 anos ronda os 6 itens.

De acordo com o modelo de memória de trabalho, proposto por Baddeley e Hitch (1974), a amplitude de memória não caracteriza o seu aspecto dinâmico. Por exemplo, quando comparada com uma tarefa mais complexa de amplitude de leitura, em que é avaliado o número de palavras recordadas durante a leitura de frases, a amplitude de memória é considerada um fraco preditor das competências de leitura. Os testes de memória de trabalho são idênticos às tarefas de amplitude de memória para dígitos na medida em que também é pedido aos participantes para repetirem pela mesma ordem os itens apresentados. Diferem contudo destes pois os participantes simultaneamente têm que desempenhar outra tarefa que consiste em transformar temporariamente a informação que se encon- tra no armazenamento a curto prazo. Por exemplo, no caso da tarefa de amplitude de leitura os participantes processam informação nova através da leitura de frases enquanto tentam reter a sequência das últimas palavras das frases lidas. No geral, apesar do nível absoluto de desempenho nos testes de memória de trabalho ser mais reduzido do que nas tarefas de amplitude de memória para dígitos, observa-se o mesmo aumento de desempenho consoante a idade.

O modelo de memória de trabalho, tal como foi concebido por Baddeley e Hitch (1974), define-se por um modelo tripartido que comporta um sistema denominado executor central, responsável pelas funções reguladoras incluindo a atenção, o controle da acção e a resolução de problemas. Os outros dois componentes, loop fonológico e o bloco de notas visuo-espacial, são sistemas especializados que retêm e manipulam a informação codificada respectivamente de forma fonológica e visual/espacial.

O loop fonológico é constituído por um armazenamento fonológico de curto prazo que mantém a informação verbal e um processo de recapitulação sub-vocal. Se a recapitulação não ocorre, a informação verbal rapidamente decai. O aumento na taxa de recapitulação com a idade tem sido considerado o principal factor de desenvolvimento da capacidade da memória a curto prazo pois permite que maior quantidade de informação verbal seja mantida no registo fonológico. De acordo com Gathercole e Hitch (1993) o início do processo de recapitulação espontânea ocorre apenas por volta dos 7 anos. Estes autores consideram que antes dessa idade, a capacidade do loop fonológico corresponde à capacidade do seu armazenamento. Enquanto que nos adultos as taxas de articulação (recapitulação sub-vocal) e as suas amplitudes de memória imediata estão fortemente associadas, por volta dos 4 anos não foi encontrada associação entre estas duas variáveis (Gathercole, Adams \& Hitch, 1994). Este resultado sugere que os pré-escolares não usam a recapitulação sub-vocal como meio de manter o material verbal no armazenamento fonológico.

Contudo, a recapitulação não pode ser considerado o único factor de evolução da memória de trabalho pois a amplitude de memória aumenta mesmo nos anos prévios à sua utilização. A amplitude de memória aumenta nas crianças mais novas provavelmente porque, com a idade, também aumenta a velocidade com que os itens são articulados na evocação, o que implica uma redução no declínio dos itens mantidos no armazenamento fonológico (Gathercole, 1998). Factores como o conhecimento do léxico e da estrutura probabilística das combinações do som na linguagem parecem também contribuir para as mudanças que ocorrem no desempenho em testes de memória imediata (Gathercole).

O bloco de notas visuo-espacial é o outro componente da memória de trabalho que se subdivide num componente visual e noutro espacial, e que contém e manipula mentalmente as características físicas dos estímulos, tais como a forma, a cor e o movimento. Em relação ao funcionamento deste componente ocorre uma mudança desenvolvi- 
mental importante também por volta dos 7 anos de idade. As crianças mais novas comparativamente às mais velhas ou adultos são mais dependentes deste componente em tarefas de memória imediata para material visual. O estudo de Hitch, Halliday, Schaafstal e Schraagen (1988) indica que em tarefas em que os objectos a recordar partilham muitas características físicas (ex., caneta, garfo, pente, chave) as crianças de 5 anos têm mais dificuldade em recordá-los, relativamente a objectos que partilham poucas características físicas (ex., boneca, banheira, luva, sabonete). As crianças de 10 anos, por sua vez, não são sensíveis à similaridade física dos objectos, mas apresentam menores níveis de recordação quando os objectos possuem um nome mais comprido (ex., umbrella, kangaroo, policeman, banana). Este estudo sugere que as crianças mais novas dispõem apenas do bloco de notas visuo-espacial para recordar as qualidades físicas dos estímulos provavelmente porque não são capazes de gerar códigos fonológicos para os itens visuais, enquanto que as crianças mais velhas adoptam a estratégia de recordar as figuras sob forma verbal, funcionando o loop fonológico como mediador do desempenho na tarefa de memória visual. A partir dos 7 anos, quando possível, as crianças usam a recapitulação para codificar os inputs visuais numa forma fonológica. Quando o material não é passível de ser registado fonologicamente ainda não há consenso relativo à explicação do aumento de desempenho nos testes de bloco de notas visuo-espacial com a idade. É possível que esteja relacionado com mudanças na capacidade do próprio armazenamento, com a aplicação de estratégias mais eficazes ou ainda com o maior apoio do executor central (Gathercole, Pickering, Benjamin, \& Hannah, 2004).

De forma a obter uma avaliação do desenvolvimento da memória visuo-espacial é conveniente usar tarefas de memória em que os estímulos não possam ser facilmente reconvertidos para a forma fonológica. A tarefa de memória para padrões é um exemplo deste tipo de tarefas e consiste em detectar a localização da(s) forma(s) que falta $(\mathrm{m})$ num padrão de quadrados anteriormente apresentados, alguns dos quais estavam preenchidos com diferentes tipos de formas. Através deste tipo de tarefa, a maioria dos estudos indica um aumento regular da amplitude de memória visual entre os 5 e os 11 anos (Gathercole, 1998).

O executor central exerce as funções reguladoras e de controlo das actividades, especificamente controlando o fluxo de informação na memória de trabalho, recuperando material dos sistemas de memória a longo prazo e ainda controlando e planeando a acção. O desenvolvimento deste componente da memória de trabalho tem sido analisado através de tarefas complexas que requerem o armazenamento da informação e a transformação do material, tais como tarefas de amplitude de leitura e escuta e de memória para dígitos em sentido inverso. A primeira consiste em, através da leitura de frases ou da escuta, processar essa informação e ao mesmo tempo reter a sequência das últimas palavras das frases lidas ou ouvidas. A segunda tarefa consiste em evocar os dígitos apresentados na ordem inversa à sua apresentação. Apesar dos estudos serem limitados parece existir um aumento do desempenho com a idade em tarefas complexas de memória de trabalho (Gathercole, 1998). Por exemplo, o estudo de Siegel (1994) indica que existe um aumento regular na amplitude de escuta entre os 6 e os 15 anos de idade.

De acordo com Gathercole et al. (2004), a estrutura modular básica da memória de trabalho parece já estar formada aos 6 anos, ou até mais cedo, mas a capacidade de cada componente aumenta linearmente até à adolescência. A organização estrutural da memória de trabalho mantém-se constante com a idade, observando-se no entanto uma ligação mais próxima do executor central com o loop fonológico e com o bloco de notas visuo-espacial ao longo do desenvolvimento, mas permanecendo estes dois últimos componentes relativamente independentes entre si.

Para além do modelo de Baddeley (1986) existem outras perspectivas sobre a forma como a memória de trabalho funciona, as quais não implicam uma divisão tripartida e acarretam diferenças no tipo de mudanças desenvolvimentais consideradas. Por exemplo, no modelo proposto por N. Cowan (1997) o foco de atenção é considerado um aspecto impor tante da memória de trabalho. $\mathrm{O}$ foco de atenção é, por um lado, controlado pelo executor central quando representa o processamento voluntário de estratégias e, por outro, por mudanças automáticas de atenção relativas a modificações abruptas do estímulo. De acordo com este modelo diferenças desenvolvimentais da memória de trabalho provêm de um funcionamento distinto do foco de atenção. Estas diferenças desenvolvimentais referem-se à quantidade de material que pode ser captado pelo foco de atenção e implicam que quanto maior for a quantidade de informação atendida mais informação será processada. Referem-se ainda à eficiência com que a atenção capta os estímulos relevantes e como a atenção pode ser usada para suprimir ou inibir a activação de estímulos irrelevantes. Quando o espaço disponível do armazenamento é tomado por informação irrelevante que não foi devidamente suprimida a memória de trabalho torna-se menos eficiente. De acordo com esta perspectiva o desenvolvimento da memória de trabalho ocorre sobretudo porque as crianças mais velhas são mais eficientes em focar a atenção na informação relevante e em inibirem aspectos irrelevantes que, muitas vezes, são activados automaticamente (N. Cowan, 1997).

\section{Memória Declarativa ou Explícita}

Um dos temas de interesse do desenvolvimento da memória a longo prazo refere-se à determinação do momento em que surge a memória declarativa ou explícita. Schacter e Moscovitch (1984) propuseram que a memória dos bebés até aproximadamente um ano era considerada procedimental e que a partir dessa idade emergia um novo sistema de memória designado de memória explícita. Estes autores compararam as competências de memória dos bebés com as capacidades mnésicas observadas nos amnésicos e sugeriram que, em ambos, a memória explícita estava ausente enquanto que a memória procedimental 
permanecia intacta. A ausência de memória explícita, de acordo com estes autores, reflectia-se num desempenho pobre em tarefas que são mediadas por um sistema que requer consciência. Esta ideia tem trazido muita polémica e reacções de alguns investigadores (ex, Bauer, 1996; Meltzoff, 1995), os quais defenderam que os bebés já demonstram algumas formas de memória explícita antes de 1 ano, tais como a evocação através de imitação diferida (para uma revisão destes estudos ver Bauer, 2002). Embora esta seja uma questão muito debatida dentro do tema do desenvolvimento da memória ela escapa das fronteiras deste artigo por se referir a uma faixa etária inferior a 3 anos de idade e, consequentemente, implicar uma memória não verbal.

Apesar de ser inquestionável que a partir dos 3 anos as crianças já possuem uma memória considerada explícita ela evolui, sofre alterações e com a idade vai dando origem a novas formas de memória. Passaremos então a identificar os aspectos da memória declarativa ou explícita que sofrem mais alterações com o desenvolvimento.

A transferência de informação da memória a curto prazo para a memória a longo prazo tem sido estudada através dos efeitos da posição dos itens em tarefas de evocação livre. Quando são apresentadas palavras não relacionadas e, a seguir os participantes são convidados a evocá-las em qualquer ordem, verifica-se uma tendência robusta para as primeiras e as últimas palavras da lista serem as melhores recordadas. A estes efeitos chama-se respectivamente efeito de primazia, proveniente da recuperação da memória a longo prazo, e efeito de recência, proveniente da recuperação da memória a curto prazo. Bjorklund e Muir (1988) relataram as diferenças existentes nas curvas de posição serial de crianças de 6, 9 e 14 anos. O efeito de recência é observável em qualquer destas idades, existindo diferenças desenvolvimentais mínimas na porção da curva serial que reflecte este efeito. A diferença etária é mais acentuada no que se refere ao efeito de primazia. Este efeito vai aumentando com a idade, sendo praticamente inexistente aos 6 anos de idade. Segundo Bjorklund e Muir a evidência de um efeito de primazia mais acentuado nos mais velhos reflecte o uso de estratégias de memória.

A avaliação da memória declarativa ou explícita é normalmente realizada através de tarefas de evocação, evocação guiada e reconhecimento. As maiores diferenças etárias observam-se sobretudo no desempenho das tarefas de evocação. Autores como Perlmutter (1984) sugerem que isto se deve ao facto da evocação ser mais sensível ao uso de estratégias de memória e, consequentemente, esta tarefa é capaz de distinguir as crianças que já utilizam as estratégias daquelas que ainda não as usam de forma espontânea.

De uma forma geral, as crianças pré-escolares possuem um bom desempenho em tarefas de reconhecimento mas, a maior parte das vezes, apresentam níveis baixos em tarefas de evocação (Perlmutter \& Lange, 1978). O facto de existirem pequenas diferenças etárias relativamente ao reconhecimento sugere que as crianças mais novas são capazes de codificar correctamente a informação. Por ou- tro lado, ao demonstrarem uma evocação pobre significa que estas crianças não são hábeis na procura das suas memórias ou que necessitam de ajuda, através de pistas, para efectuar essa procura com sucesso (Schneider \& Bjorklund, 1998).

Para alguns tipos específicos de reconhecimento a diferença de desempenho entre idades distintas chega a ser nula. É o caso do reconhecimento da localização espacial dos itens, medido quer através de tarefas de reconstrução ou de jogos como o "Memória”, em que as crianças necessitam de reter a localização espacial de figuras. Schneider e Bjorklund (1998) sugerem que as crianças mais novas apresentam um desempenho invulgar neste tipo de tarefa porque o reconhecimento espacial baseia-se sobretudo em capacidades visuo-espaciais que se desenvolvem cedo, não necessitando de competências da memória verbal. A contrastar com o desempenho obtido através de tarefas de reconhecimento visuo-espacial, em tarefas de memória verbal assiste-se a desempenhos díspares de acordo com a idade (Schumann-Hengsteler, 1992).

Quanto ao desenvolvimento do desempenho em tarefas de evocação guiada comparativamente à evocação simples, o estudo de Ceci e Howe (1978), utilizando listas de palavras, revelou que crianças de 4, 7 e 10 anos obtiveram níveis de evocação semelhantes num teste imediato de evocação guiada, mas grandes diferenças em tarefas de evocação livre realizadas com um dia de intervalo. Este tipo de estudos apoia a interpretação de que o uso espontâneo de estratégias é o elemento que distingue o diferente desempenho das crianças em tarefas de evocação livre.

Até agora reportámo-nos à evolução da memória de longo prazo relativa à apresentação de itens, sejam estes palavras ou figuras. Embora tenha sido desenvolvida menos investigação referente à memória sobre a própria experiência dos sujeitos, a maioria destes estudos indica também uma evolução da memória para acontecimentos.

A memória autobiográfica foi proposta como sendo originária da evolução da memória para acontecimentos e corresponde à memória em sentido restrito, ou episódica, tal como foi definida por Tulving (1985). Nelson (1993) refere que a definição de memória episódica proposta por Tulving é mais restrita do que o sistema proposto por Schacter e Moscovitch (1984). A consciência de que algo se passou (Schacter e Moscovitch) não equivale a que algo se passou em determinado sítio e determinado lugar (Tulving). Nelson sugere que este último estado de consciência implica a existência daquilo que ele designou de memória autobiográfica. Supõe-se que surja por volta dos 3/4 anos (Nelson, 1992) quando, na terminologia de Perner (2000), a criança já consegue manter duas representações simultaneamente, o self passado e o self presente. Nesta perspectiva, a memória autobiográfica corresponde a um tipo de memória episódica que pode ser acedida posteriormente e que faz parte da nossa história (M. A. Cowan, 1990). Para Nelson (1992) o surgimento deste tipo de memória envolve mudanças na função da memória que põem termo ao período correspondente à amnésia infantil, ou seja, à incapacidade que nós possuímos, enquanto 
adultos, em recordar episódios dos primeiros anos de vida. Os estudos realizados com idosos sobre a distribuição de memórias ao longo do ciclo de vida (ex., Rubin, 2000, 2002) revelam, em média, uma escassa percentagem de $2.2 \%$ de memórias obtidas antes da idade dos 3 anos. Estes estudos evidenciam uma explosão de memórias para acontecimentos que ocorreram entre os 10 e os 30 anos, um declínio correspondente à idade dos 45-50 anos e um novo aumento de memórias referentes aos últimos anos de vida.

Para que a memória autobiográfica seja estabelecida tem de ocorrer uma evolução da memória episódica na forma como as memórias estão organizadas, armazenadas e são recuperadas. Nelson e Fivush (2000, 2004) atribuem à linguagem um papel de primordial importância para que ocorra esta evolução pois, a linguagem não só ajuda a formar representações organizadas das experiências passadas, como torna consciente que as memórias são representações de acontecimentos passados. Nesta perspectiva sociolinguística (Fivush, 2002; Nelson \& Fivush, 2004), a capacidade de partilhar a nossa experiência passada com os outros por meio da linguagem proporciona, por um lado, o surgimento da memória autobiográfica e, por outro, implica o fim do período da amnésia infantil. Apesar de esta perspectiva ser considerada das mais influentes para explicação do fenómeno da amnésia infantil, não tem sido consensualmente aceite e a resolução desta questão continua por esclarecer (ver também outras perspectivas recentes apresentadas por Wang, 2003).

Contrastando com a perspectiva de Nelson (1993), Howe e Courage (1997) sugerem que não existe uma descontinuidade nos sistemas de memória antes e após o aparecimento da memória autobiográfica e que esta surge mais cedo do que Nelson $(1992,1993)$ propôs. De acordo com Howe e Courage (1997) a memória autobiográfica emerge entre os 18 e os 24 meses, quando a criança começa a ter a consciência de si própria como entidade possuidora de competências cognitivas específicas. Nesta perspectiva (Howe, Courage, \& Edison, 2003), os sistemas básicos de memória já existem na infância, mas a memória autobiográfica começa a operar quando, na terminologia dos autores, se constrói o self cognitivo. Assim, a memória autobiográfica surge quando esta estrutura do conhecimento já permite à criança organizar as memórias das experiências que ocorrem ao próprio.

Através de diários escritos pelas mães e por entrevistas às próprias crianças, Nelson (1993) estudou a organização da memória autobiográfica em diferentes idades. Numa primeira fase em que parecem ocorrer representações difusas, as crianças têm dificuldade em relatar memórias específicas de acontecimentos recorrentes sem a ajuda de pistas de recuperação. A informação encontra-se organizada em termos de roteiros (scripts) ou esquemas (schemas) arranjados sequencialmente. O termo script, proveniente de Schank e Abelson (1977), refere-se a um tipo de memória geral que reflecte a estrutura do acontecimento familiar, representado através de sequências de acções organizadas espacial e temporalmente. Na medida em que se refere a representações de acontecimentos gerais, o script é definido como uma das primeiras formas de memória semântica derivada da experiência (Nelson \& Fivush, 2000). O exemplo típico de um script corresponde a uma ida a um restaurante que inclui determinadas acções sequenciais como por exemplo: entrar, sentar-se, ler o menu, fazer o pedido, receber a comida, comer, pedir a conta, pagar e sair. Devido ao facto de possuírem mais experiência dos acontecimentos e uma linguagem mais complexa, os scripts das crianças mais velhas, comparativamente com os das mais novas, são de uma forma geral mais elaborados, incluindo mais acções constituintes do acontecimento, mais alternativas (ex., relativo ao script "ida ao McDonald's" - "pode-se encomendar um hamburguer ou um cheeseburguer"), mais actividades condicionais (ex., "se não quiser sair do carro a encomenda é feita através do carro") e mais actividades opcionais (ex., "pode-se pedir sobremesa") (Fivush, 1997).

As crianças, mesmo as mais novas, possuem representações bem organizadas de acontecimentos familiares, em que normalmente as acções constituintes dos acontecimentos são relatadas numa sequência temporal apropriada (Fivush, 1997). No entanto, quando uma experiência se repete, os detalhes específicos do episódio tendem a confundir-se com outros episódios do mesmo acontecimento e as crianças mais novas não são capazes de recuperar um episódio de uma experiência familiar. As crianças tendem a recordar os acontecimentos familiares recorrentes em termos de roteiros ou esquemas familiares, porém, elas são capazes de relatar memórias específicas se os acontecimentos não são familiares. Por exemplo, o estudo de Hamond e Fivush (1991) analisou a recordação de uma visita ao DisneyWorld efectuada meses ou um ano após a sua realização. Mesmo as crianças mais novas foram capazes de recordar as suas experiências numa quantidade semelhante à recordada pelas crianças mais velhas. Desde que os acontecimentos sejam novos e relevantes para a própria criança parece que estas conseguem recordá-los por consideráveis períodos de tempo.

Hudson e Nelson (1986) referem que as crianças por volta dos 3 anos de idade fornecem relatos mais curtos, com menos informação incluída, quando lhes é perguntado "o que aconteceu em determinado dia ..." em comparação com a pergunta mais geral do tipo "o que é que normalmente acontece ...” para acontecimentos recorrentes. Também a recordação é normalmente superior para acontecimentos específicos novos comparativamente com acontecimentos específicos familiares. A ideia de que as representações de acontecimentos familiares das crianças préescolares possuem uma ordem canónica que controla a recordação é partilhada por Mandler (1990), o qual observou que as crianças mais novas recordam melhor os acontecimentos quando o material é apresentado numa sequência correcta e natural em comparação com a apresentação desordenada dos acontecimentos. As crianças mais novas parecem estar mais dependentes de esquemas familiares para recuperar a informação, contrastando com os adultos, os quais são mais flexíveis e possuem mais opções para a recuperação. 
De acordo com Nelson (1993), a primeira evolução da memória episódica fornece à criança a capacidade de recuperar um episódio específico de um acontecimento recorrente. Outro desenvolvimento importante no caminho da memória autobiográfica refere-se à capacidade de recuperar memórias na ausência de pistas externas. As crianças mais novas necessitam de pistas específicas para conseguirem recordar detalhes sobre acontecimentos particulares, enquanto que nas crianças mais velhas a recordação é espontânea, sem necessidade de existência de pistas. Para este autor esta aquisição é essencial para o estabelecimento da memória autobiográfica e confere à memória a possibilidade de reexperienciar o passado através da consciência autonoetica (Nelson \& Fivush, 2004).

\section{Memória Não Declarativa ou Implícita}

Os estudos que analisam o desenvolvimento da memória implícita têm utilizado sobretudo material pictórico, em substituição de listas de palavras, de forma a poderem incluir crianças que ainda não dominam a leitura. Dentro das tarefas de identificação perceptiva, a prova de identificação de figuras fragmentadas tem sido das mais usadas pelos investigadores. Nesta tarefa, o efeito de memória implícita é obtido quando, após as crianças terem sido expostas a determinadas figuras, conseguem mais facilmente identificá-las a partir de fragmentos, comparativamente à identificação de outras figuras fragmentadas que não tinham sido visualizadas antes.

O estudo de Parkin e Streete (1988) foi dos primeiros a ser realizado nesta área. Estes autores testaram crianças com 3, 5, 7 anos e adultos numa tarefa de identificação de fragmentos de figuras e numa tarefa de reconhecimento e concluíram que o reconhecimento aumentou com a idade enquanto a facilitação na identificação das figuras (priming) manteve-se estável, quando calculada numa proporção relativa à aprendizagem inicial.

De uma forma geral, estes resultados têm sido corroborados pela maioria dos estudos que usaram a prova de identificação de fragmentos de figuras (Anooshian, 1997; Carneiro \& Esteves, 2001; Drummey \& Newcombe, 1995; Hayes \& Hennessy, 1996; Perez, Peynircioglu, \& Blaxton, 1998; Russo, Nichelli, Gilbertoni, \& Cornia, 1995). Também quando é empregue material verbal, tal como na prova de identificação de palavras fragmentadas, a quantidade de priming analisada de forma proporcional, tende a manter-se estável ao longo da idade (Komatsu, Naito, \& Fuke, 1996; Naito, 1990).

Relativamente aos resultados obtidos com tarefas perceptivas de memória implícita, tais como as tarefas de identificação de figuras ou palavras fragmentadas, em que o desempenho dos sujeitos é dependente das características físicas dos estímulos, parece existir consenso relativamente à invariabilidade da memória implícita. No entanto, quando as tarefas enfatizam as relações semânticas entre os itens, os resultados das investigações não têm sido inteiramente consistentes. Atendendo a que requerem um processamento conceptual, poder-se-iam esperar diferenças etárias no priming conceptual.
Poucos estudos têm sido desenvolvidos com o intuito de analisar esta evolução da memória implícita através de provas conceptuais. No estudo de Greenbaum e Graf (1989) foi utilizada uma prova de memória implícita conceptual denominada de tarefa de produção de exemplares de categorias. Esta prova consistiu em expor crianças de 3 e 5 anos a figuras de animais do Jardim Zoológico e passado algum tempo, solicitar-lhes a produção de exemplares que pertenciam à categoria anteriormente apresentada e a outra categoria que não tinha sido apresentada antes. Os autores mediram o priming conceptual através da diferença entre a quantidade de exemplares produzidos para os dois tipos de categorias. Os resultados revelaram efeitos de priming invariáveis com a idade, apesar de se ter verificado um melhor desempenho dos mais velhos numa tarefa semelhante de evocação. Também o estudo de Carneiro e Esteves (2001), no qual foi utilizado o mesmo tipo de tarefa implícita conceptual, corroborou estes resultados. No entanto, ao ser analisado o priming para exemplares típicos e atípicos das categorias, os estudos de Mecklenbrauker, Hupbach e Wippich (2003) e de Murphy, McKone e Slee (2003) revelaram existir uma evolução da memória implícita conceptual para exemplares atípicos de categorias taxonómicas familiares, sem contudo serem observadas diferenças relativamente aos elementos típicos.

Outros estudos manipularam o tipo de processamento imposto ao sujeito através das instruções da tarefa (Hayes \& Hennessy, 1996; Komatsu et al., 1996; Naito, 1990; Perez et al., 1998). Quando se pretendia que fosse adoptado um processamento perceptivo, era pedido aos participantes para prestarem atenção às características físicas do estímulo (ex., contar o número de letras que compõem a palavra alvo), enquanto que para adoptarem um processamento conceptual era-lhes solicitado que atendessem às características semânticas do mesmo (ex., gerar um sinónimo da palavra alvo). Na generalidade, os estudos que utilizaram este tipo de manipulação mostraram, em ambos os tipos de instrução, uma estabilidade da memória implícita ao longo da idade, enquanto que nas provas explícitas (evocação ou reconhecimento) o desempenho foi superior tanto para o grupo de crianças mais velhas como para o processamento conceptual. No entanto, nem todos os estudos foram concordantes. Komatsu e colaboradores (1996) não obtiveram diferenças de priming entre crianças de 7, 11 anos e adultos numa tarefa de identificação de fragmentos de palavras na condição em que foi utilizado um processamento perceptivo, mas demonstraram existir maior quantidade de priming proporcional nos participantes mais velhos quando estes enveredaram por um processamento conceptual.

Em suma, a investigação realizada até ao momento no âmbito do desenvolvimento da memória implícita revela uma estabilidade do priming quando testado a nível perceptivo, apesar da evidente evolução da memória explícita. Quando, por sua vez, o priming é testado a nível conceptual os dados não são conclusivos, possivelmente devido às diferentes metodologias adoptadas nos estudos. Presentemente tem sido proposta uma forma alternativa 
de interpretação dos resultados nesta área (Mecklenbrauker et al., 2003; Murphy et al., 2003). Em vez desta dissociação desenvolvimental ser atribuída aos diferentes sistemas de memória ou ao tipo de processamento (perceptivo ou conceptual), tem sido enfatizado o papel do processamento estratégico e do conhecimento de base como possíveis determinantes das diferenças encontradas ao longo do desenvolvimento. Enquanto que é atribuído um papel fundamental no desenvolvimento da memória explícita ao processamento estratégico, o conhecimento de base pode estar implicado em ambos os tipos de memória. Mecklenbrauker e colaboradores (2003) e Murphy e colaboradores (2003) defendem que a memória implícita pode apresentar diferenças com a idade se o conhecimento de base relativo ao domínio testado também se encontra em evolução. Estes autores explicam as diferenças etárias observadas na memória implícita conceptual quando são estudados os exemplares atípicos das categorias pelo facto das crianças mais velhas, em comparação com os pré-escolares, possuírem um conhecimento de base mais rico no que se refere às relações categoriais para exemplares atípicos de categorias. Por sua vez, a invariabilidade obtida para o priming relativo aos elementos típicos foi justificada por as relações categoriais para elementos típicos apresentarem menores divergências etárias.

\section{Conclusão}

Tendo em consideração os diferentes armazenamentos ou sistemas de memória, verificam-se mudanças quantitativas e qualitativas importantes ao longo do desenvolvimento da memória de trabalho e explícita. Contudo, para a memória não declarativa ou implícita parecem não ocorrer evoluções na quantidade de priming, pelo menos quando este é testado a nível perceptivo. Supõe-se que dado a falta de resultados conclusivos relativamente ao desenvolvimento da memória implícita conceptual surjam, brevemente, mais estudos que possam fornecer respostas esclarecedoras quanto à evolução da memória implícita conceptual.

\section{Referências}

Anooshian, L. J. (1997). Distinctions between implicit and explicit memory: Significance for understanding cognitive development. International Journal of Behavioral Development, 21, 453-478.

Atkinson, R. C., \& Shiffrin, R. M. (1968). Human memory: A proposed system and its control processes. In K. W. Spence \& J. T. Spence (Eds.), Psychology of learning and motivation: Vol. 2. New York: Academic Press.

Baddeley, A. D. (1986). Working memory. Oxford, UK: Clarendon. Baddeley, A. D., \& Hitch, G. J. (1974). Working memory. In G. Bower (Ed.), The psychology of learning and motivation: Advances in research and theory (Vol. 8, pp. 47-90). New York: Academic Press.

Bauer, P. (1996). Recalling past events: From infancy to early childhood. Annals of Child Development, 11, 25-71.

Bauer, P. (2002). Early memory development. In U. Goswami
(Ed.) Blackwell handbook of childhood cognitive development (pp. 127-146). Malden, MA: Blackwell.

Bjorklund, D. F., \& Muir, J. E. (1988). Children's development of free recall memory: Remembering on their own. Annals of Child Development, 5, 79-123.

Carneiro, M. P., \& Esteves, F. (2001). Dissociação entre o desenvolvimento da memória explícita e implícita em crianças. Psicologia, Educação e Cultura, 5, 195-2 16.

Ceci, S. J., \& Howe, M. J. (1978). Age-related differences in recall as a function of retrieval flexibility. Journal of Experimental Child Psychology, 26, 432-442.

Cohen, N. J., \& Squire, L. R. (1980). Preserved learning and retention of pattern-analyzing skill in amnesia: Dissociation of knowing how and knowing what. Science, 210, 207-210.

Cowan, M. A. (1990). Autobiographical memory: An introduction. Milton Keynes, UK: Open University Press.

Cowan, N. (1997). The development of working memory. In N. Cowan (Ed.), The development of memory in childhood (pp. 113137). Hove, UK: Psychology Press.

Dempster, F. N. (1981). Memory span: Sources of individual and developmental differences. Psychological Bulletin, 89, 63-100.

Drummey, A. B., \& Newcombe, N. (1995). Remembering versus knowing the past: Children's explicit and implicit memories for pictures. Journal of Experimental Child Psychology, 59, 549-565

Fivush, R. (1997). Event memory in early childhood. In N. Cowan (Ed.), The development of memory in childhood (pp. 113137). Hove, UK: Psychology Press.

Fivush, R. (2002). Children's long-term memory of childhood events. In P. Graf \& N. Ohta (Eds.), Lifespan development of human memory (pp. 83-100). Cambridge, MA: MIT Press.

Gardiner, J. M. (1988). Functional aspects of recollective experience. Memory \& Cognition, 16, 309-313.

Gardiner, J. M., \& Java, R. I. (1993). Recognising and remembering. In A. Collins, S. E. Gathercole, M. A. Conway $\&$ P. E. Morris (Eds.), Theories of memory (pp. 163-188). Hove, UK: Erlbaum

Gathercole, S. E. (1998). The development of memory. Journal of Child Psychology and Psychiatry, 39, 3-27.

Gathercole, S. E., Adams, A., \& Hitch, G. J. (1994). Do young children rehearse? An individual-differences analysis. Memory \& Cognition, 22, 201-207.

Gathercole, S. E., \& Hitch, G. J. (1993). Developmental changes in short-term memory: A revised working memory perspective. In A. Collins, S. E. Gathercole, M. A. Conway, \& P. E. Morris (Eds.), Theories of memory (pp. 189-209). Hove, UK: Erlbaum.

Gathercole, S. E., Pickering, S. J., Benjamin, A., \& Hannah, W. (2004). The structure of working memory from 4 to 15 years of age. Developmental Psychology, 40, 177-190.

Graf, P., \& Schacter, D. L. (1985). Implicit and explicit memory for new associations in normal and amnesics subjects. Journal of Experimental Psychology: Learning, Memory and Cognition, 11, 386-396.

Greenbaum, J. L., \& Graf, P. (1989). Preschool period development of implicit and explicit remembering. Bulletin of the Psychonomic Society, 27, 417-420.

Hamond, N. R., \& Fivush, R. (1991). Memories of Mickey Mouse: Young children recount their trip to Disneyworld. Cognitive Development, 6, 433-448.

Hayes, B. K., \& Hennessy, R. (1996). The nature and development of nonverbal implicit memory. Journal of Experimental Child Psychology, 63, 22-43

Hitch, G. J., Halliday, M. S., Schaafstal, A. M., \& Schraagen, J. M. C. (1988). Visual working memory in young children. 
Memory \& Cognition, 16, 120-132.

Hoving, K. L., Spencer, T., Robb, K. Y., \& Schulte, D. (1978). Developmental changes in visual information processing. In P. A. Ornstein (Ed.), Memory development in children. Hillsdale, NJ: Erlbaum.

Howe, M. L., \& Courage, M. L. (1997). The emergence and early development of autobiographical memory. Psychological Review, 104, 499-523.

Howe, M. L., Courage, M. L., \& Edison, S. C. (2003). When autobiographical memory begins. Developmental Review; 23, 471-494.

Hudson, J., \& Nelson, K. (1986). Repeated encounters of a similar kind: Effects of familiarity on children's autobiographical memory. Cognitive Development, 1, 253-272.

Komatsu, S., Naito, M., \& Fuke, T. (1996) Age-related and intelligence-related differences in implicit memory: Effects of generation on a word-fragment completion test. Journal of Experimental Child Psychology, 62, 151-172

Mandler, J. M. (1990). Recall and its verbal expression. In R. Fivush \& J. Hudson (Eds.), Knowing and remembering in young children (pp. 3 17-330). New York: Cambridge University Press.

Mecklenbrauker, S., Hupbach, A., \& Wippich, W. (2003). Agerelated improvements in a conceptual implicit memory test. Memory \& Cognition, 31, 1208-1217.

Meltzoff, A. N. (1995). What infant memory tells us about infantile amnesia: Long-term and deferred imitation. Journal of Experimental Child Psychology, 59, 497-515.

Miller, G. A. (1956). The magic number seven, plus or minus two: Some limits on our capacity for processing information. Psychological Review; 63, 81-97.

Murphy, K., McKone, E., \& Slee, J. (2003). Dissociations between implicit and explicit memory in children: The role of strategic processing and the knowledge base. Journal of Experimental Child Psychology, 84, 124-165.

Naito, M. (1990). Repetition priming in children and adults: Agerelated dissociation between implicit and explicit memory. Journal of Experimental Child Psychology, 50, 462-484

Nelson, K. (1992). Emergence of autobiographical memory at age 4. Human Development, 35, 172-177.

Nelson, K. (1993). Explaining the emergence of autobiographical memory in early childhood. In A. Collins, S. E. Gathercole, M. A. Conway \& P. E. Morris (Eds.), Theories of memory (pp. 355-385). Hove, UK: Erlbaum.

Nelson, K., \& Fivush, R. (2000). Socialization of memory. In E. Tulving \& F. I. M. Craik (Eds.), The Oxford handbook of memory (pp. 283-295). Oxford, UK: Oxford University Press.

Nelson, K., \& Fivush, R. (2004). The emergence of autobiographical memory: A social cultural developmental theory. Psychological Review, 111, 486-511.

Parkin, A. J. (1997). Memory and amnesia (2nd. ed.). Oxford, UK: Blackwell.

Parkin, A. J., \& Streete, S. (1988) Implicit and explicit memory in young children and adults. British Journal of Psychology, 79, 361-369

Perez, L. A., Peynircioglu, Z. F., \& Blaxton, T. A. (1998) Developmental differences in implicit and explicit memory performance. Journal of Experimental Child Psychology, 70, 167185

Perlmutter, M. (1984). Continuities and discontinuities in early human memory paradigms, processes and performance. In R. Kail \& N. E. Spear (Eds.), Comparative perspectives on the development of memory (pp. 253-284). Hillsdale, NJ: Erlbaum.

Perlmutter, M., \& Lange, G.A. (1978). A developmental analysis of recall-recognition distinctions. In P. A. Ornstein (Ed.),
Memory development in children (pp. 243-258). Hillsdale, NJ: Erlbaum.

Perner, J. (2000). Memory and theory of mind. In E. Tulving \& F. I. M. Craik (Eds.), The Oxford handbook of memory (pp. 297312). Oxford, UK: Oxford University Press.

Roediger, H. L., \& McDermott, K. B. (1993). Implicit memory in normal human subjects. In F. Boller \& J. Grafman (Eds.), Handbook of Neuropsychology: Vol. 8 (pp. 63-131). Amsterdam, Netherlands: Elsevier.

Rubin, D. C. (2000). The distribution of early childhood memories. Memory, 8, 265-269.

Rubin, D. C. (2002). Autobiographical memory across the lifespan. In P. Graf \& N. Ohta (Eds.), Lifespan development of human memory (pp. 159-184). Cambridge, MA: MIT Press.

Russo, R., Nichelli, P., Gibertoni, M., \& Cornia, C. (1995). Developmental trends in implicit and explicit memory: A picture completion study. Journal of Experimental Child Psychology, 59, 566-578

Schacter, D. L. (1987). Implicit memory: History and current status. Journal of Experimental Psychology: Learning, Memory, and Cognition, 13, 501-518.

Schacter, D. L. \& Moscovitch, M. (1984). Infants, amnesics, and dissociable memory systems. In M. Moscovitch (Ed.), Infant memory (pp. 173-2 16). New York: Plenum.

Schank, R., \& Abelson, A. (1977). Scripts plans goals and understanding: Hillsdale, NJ: Erlbaum.

Schneider, W., \& Bjorklund, D. (1998). Memory. In D. Kuhn, R. Siegler (Eds.) \& W. Damon (Series Ed.), Cognition, perception, and language: Vol. 2. Handbook of Child Psychology (pp. 255308). New York: Wiley.

Schumann-Hengsteler, R. (1992). The development of visuospatial memory: How to remember location. International Journal of Behavioral Development, 15, 455-471.

Siegel, L. (1994). Working memory and reading: A lifespan perspective. International Journal of Behavioural Development, 17, 109-124.

Sheingold, K. (1973). Developmental differences in intake and storage of visual information. Journal of Experimental Child Psychology, 16, 1-11

Tulving, E. (1972). Episodic and semantic memory. In E. Tulving $\&$ W. Donaldson (Eds.), The organization of memory (pp. 382403). New York: Academic Press.

Tulving, E. (1985). How many memory systems are there? American Psychologist, 40, 385-398.

Wang, Q. (2003). Infantile amnesia reconsidered: A cross-cultural analysis. Memory, 11, 65-80.

Yonelinas, A. P. (2002). The nature of recollection and familiarity: A review of 30 years of research. Journal of Memory and Language, 46, 441-517. 